\title{
Recent advances in high-throughput mass spectrometry that accelerates enzyme engineering for biofuel research
}

\author{
Lihao $\mathrm{Fu}^{1,2}$, Jianzhi Zhang ${ }^{1,2^{*}}$ and Tong $\mathrm{Si}^{1,2^{*}}$ (D)
}

\begin{abstract}
Enzymes play indispensable roles in producing biofuels, a sustainable and renewable source of transportation fuels. Lacking rational design rules, the development of industrially relevant enzyme catalysts relies heavily on highthroughput screening. However, few universal methods exist to rapidly characterize large-scale enzyme libraries. Therefore, assay development is necessary on an ad hoc basis to link enzyme properties to spectrophotometric signals and often requires the use of surrogate, optically active substrates. On the other hand, mass spectrometry (MS) performs label-free enzyme assays that utilize native substrates and is therefore generally applicable. But the analytical speed of MS is considered rate limiting, mainly due to the use of time-consuming chromatographic separation in traditional MS analysis. Thanks to new instrumentation and sample preparation methods, direct analyte introduction into a mass spectrometer without a prior chromatographic step can be achieved by laser, microfluidics, and acoustics, so that each sample can be analyzed within seconds. Here we review recent advances in MS platforms that improve the throughput of enzyme library screening and discuss how these advances can potentially facilitate biofuel research by providing high sensitivity, selectivity and quantitation that are difficult to obtain using traditional assays. We also highlight the limitations of current MS assays in studying biofuel-related enzymes and propose possible solutions.
\end{abstract}

Keywords: Biofuel, Mass spectrometry, Enzyme, High-throughput screening, Label-free analysis

\section{Introduction}

Biofuels is a type of transportation fuels derived from renewable biomass [1]. Depending on the source of biomass feedstock, biofuels are classified into three generations. The first generation of biofuels is converted from food and oil crops; the second generation is converted from lignocellulose; and the third generation is converted from algal and oleaginous microorganisms. All generations of biofuels utilize biochemical conversion at certain production stages such as biomass degradation, microbial fermentation, and lipase-mediated biodiesel synthesis [2]. Therefore, the development of efficient and robust enzyme catalysts is critical to develop economically feasible processes for biofuel production.

\footnotetext{
*Correspondence: zhangjz@siat.ac.cn; tong.si@siat.ac.cn

${ }^{1}$ Institute of Synthetic Biology, Shenzhen Institutes of Advanced Technology, Chinese Academy of Sciences, Shenzhen 518055, People's Republic of China Full list of author information is available at the end of the article
}

To identify industrially useful enzymes, large-scale protein prospecting and engineering is often required, because it is still difficult to predict enzyme properties directly from amino acid sequences [2-4]. Whereas the creation of protein homolog and mutant libraries becomes straightforward due to the advances in synthetic biology, phenotypic screening remains challenging and rate limiting [5]. Generally, ad hoc assay development is necessary to couple each enzyme property with spectrophotometric signals that are amenable to highthroughput measurement, such as cell growth, optical absorbance, and fluorescence [6]. However, such an approach is limited to a narrow range of enzyme reactions and often requires the use of expensive, surrogate substrates. To overcome these limitations, highthroughput Fourier transform infrared (FTIR) spectroscopy [7] and Raman spectroscopy [8] have been utilized in label-free optical screening, which relies on characteristic spectral features or "fingerprints" so that very

(c) The Author(s). 2020 Open Access This article is distributed under the terms of the Creative Commons Attribution 4.0 International License (http://creativecommons.org/licenses/by/4.0/), which permits unrestricted use, distribution, and 
limited structural selectivity can be achieved. Moreover, transcription factor-based biosensors that correlate product formation with the expression level of a fluorescence protein have been created to facilitate protein engineering [9-11]. Although such genetic reporters eliminate the need to develop optically active surrogate substrates, only a limited range of metabolite-sensing transcription factors are available to create such reporters.

On the other hand, mass spectrometry (MS) provides a generally applicable, label-free modality to screen enzyme libraries [12-20]. Thanks to its superior mass-resolving capabilities, MS assays provide unparalleled selectivity in assigning and quantifying various molecular species in a complex reaction mixture [13]. Furthermore, high sensitivity of MS measurement permits miniaturization of reaction volume and hence reduces screening cost. But the throughput of MS analysis is traditionally limited by a prior step of gas chromatography (GC) or liquid chromatography (LC), which often takes 5-60 min. With recent advances in instrumentation and sample preparation, time-consuming chromatographic separation can be omitted before MS analysis. Therefore, it becomes possible to apply high-throughput MS assays for enzyme screening. MS screening not only exhibits sensitivity, selectivity, and quantitation that are unattainable using traditional methods, but also greatly reduces ad hoc endeavors in assay development by providing a generally applicable platform. These combined advantages may greatly accelerate and improve the study and engineering of a wide range of enzymes. However, whereas high-throughput MS assays are increasingly used in protein research for biomedical application, they have not been widely utilized to engineer enzymes for biofuel production. This is likely due to the lack of awareness of these new MS modalities in the biofuel research communities.

In this opinion essay, we aim to introduce new highthroughput MS technologies to biofuel researchers and discuss their potential applications in engineering biofuel-related enzymes. Existing approaches and applications of protein engineering for biofuel production are reviewed elsewhere [2, 3, 21-23], primarily relying on spectrophotometric and chromatographic approaches. Although there are also review papers in literature summarizing high-throughput MS assays for protein research [12-18], the use of MS screening to study biofuel enzymes has not been covered to the best of our knowledge. Here we start with the basic MS concepts and highlight two common settings of high-throughput platforms including MALDI (matrix-assisted laser desorption/ionization) MS imaging and automated loading to an ESI (electrospray ionization) source as shown in Additional file 1 . We first summarize current screening strategies for engineering biofuel-related enzymes, and then discuss how high-throughput MS assays can provide additional advantages. We conclude with future perspectives, highlighting the potential and challenges for MSbased enzyme screening in promoting biofuel research.

\section{Basic concepts in MS and high-throughput MS approaches}

MS measures gas-phase ions generated from neutral molecules in the ion source of a mass spectrometer. These ions are separated in the mass analyzer and quantified by the ion detector. In a typical mass spectrum, relative ion abundances are plotted versus mass-tocharge $(\mathrm{m} / \mathrm{z})$ ratios, and these two values reflect quantitative and qualitative information of measured molecules, respectively. To study enzymes, two types of ion sources are often utilized, including ESI and MALDI (Fig. 1). These two sources can be coupled to various mass analyzers, such as triple quadrupole (QQQ), ion trap, time-of-flight (TOF), and Orbitrap, and these mass analyzers exhibit different detection limit, mass resolution, scan speed, and quantitation. The choice of ion sources and mass analyzers is critical to obtain chemical information of interest for a select enzyme reaction.

At any given time, molecules compete for ionization in a mass spectrometer. Abundant, easy-to-be-ionized molecules, such as buffer salts, are more readily detected and considered "contaminants". To avoid this "ion suppression" effect, GC or LC is often used to separate contaminants from target analytes, so they enter the mass spectrometer at different elution times. As chromatographic separation is time-consuming, direct sample infusion is desirable to achieve higher throughput. Here we discuss two such MS settings including MALDI MS imaging (Fig. 1a) and automated loading to ESI MS (Fig. $1 \mathrm{~b}$ and c).

MALDI MS imaging can be applied for rapid profiling of a spatially defined array of enzyme reactions on a surface called MALDI target (Fig. 1a) [12]. MALDI MS is well suited for rapid inspection of a large number of biological samples because of its simple sample preparation, high salt tolerance, and a wide coverage of diverse biomolecules [12, 25, 26]. Traditionally, macromolecules such as proteins, lipids, and glycans are the main targets for MALDI MS, but small metabolites are increasingly analyzed as well (Table 1) [32]. Various surface chemistry has been developed to immobilize and/or capture analytes on a MALDI target, allowing removal of contaminants to enhance detectability and quantitation by washing steps [12]. After matrix application, laser is applied and analyze reaction arrays at a rate of $<5 \mathrm{~s}$ per sample (Fig. 1a). When coupled with machine vision, laser sampling can be programmed to target randomly located objects such as microbial colonies [33]. This development enabled rapid engineering of multi-step enzymatic pathways using microbial cells as reaction vessels [26]. 

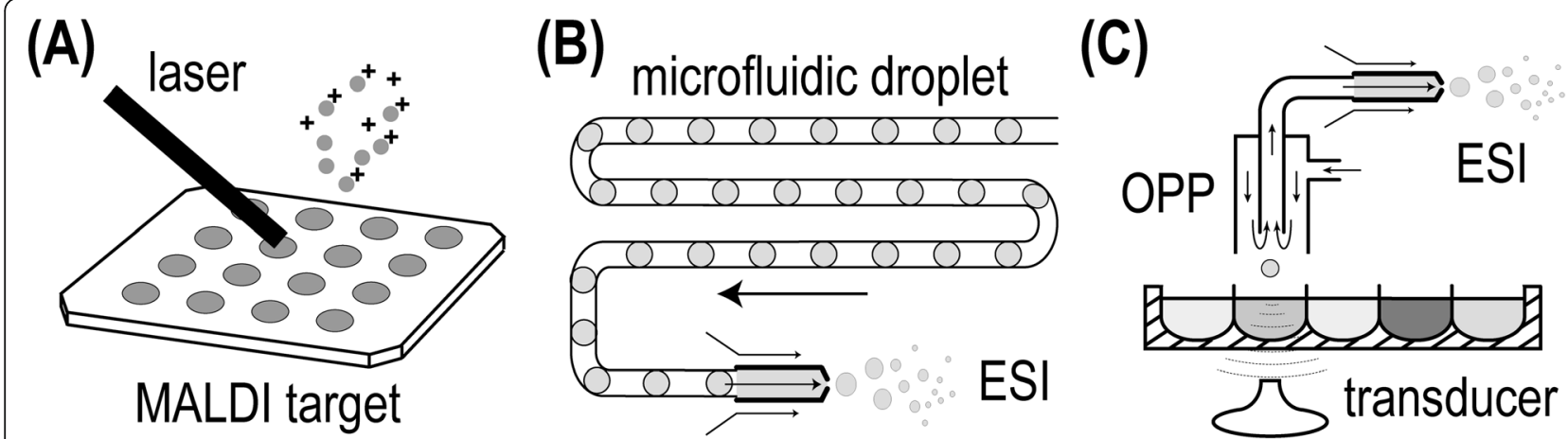

Fig. 1 High-throughput MS approaches to screen enzyme libraries. a MALDI MS imaging-based screening. Enzyme reactions are spotted as microarrays on a surface called MALDI target. Then, matrix molecules that absorb laser energy are overlaid. Laser shots are fired at high frequencies across surface microarrays for analyte desorption, ionization, and MS analysis. b Microfluidic loading to ESI MS. Enzyme reactions are performed in aqueous droplets in microfluidic channels followed by direct infusion into an ESI source. Adapted with Permission from [24]. Copyright 2018 American Chemical Society. c Acoustic loading to ESI MS. Enzyme reactions are created in microtiter plates. From each well, nanoliter droplets are ejected using an acoustic transducer. The droplets are then introduced to an ESI source via an open port probe (OPP) that carries a continuous solvent flow

ESI MS provides complementary analytical capabilities to MALDI MS. For example, small molecules $(<500 \mathrm{Da})$ are challenging targets for MALDI MS analysis due to strong matrix background signals, but they are readily detected by ESI MS (Table 1). However, ESI MS is less tolerant to contaminate interference and hence often requires LC separation. To improve throughput, solidphase extraction (SPE) can be utilized instead of LC for desalting. The Agilent RapidFire system further automates sample aspiration, SPE desalting, and ESI MS injection steps to achieve a cycling time of $\sim 10 \mathrm{~s}[27,28]$.

Alternatively, miniaturization of injected sample volume avoids "overloading" the mass spectrometer and hence reduces the impact of ion suppression. Automatic loading of small-volume samples to ESI MS can be achieved through microfluidics or acoustics (Fig. 1b and c). In a microfluidic channel, many femto- to nanoliter reactions are set up in aqueous droplets dispersed in an immiscible fluid [34]. These droplets can be directly interfaced to an ESI source for MS-based screening at a rate of $<1 \mathrm{~s}$ (Fig. 1b) [24, 29]. To achieve robust screening, it is necessary to perform systematic optimization of many parameters including flow rate, emitter configuration, and droplet-stabilizing surfactants [29]. For microfluidic droplets, it is also possible to combine optical and MS screening in a single lab-on-a-chip platform to provide complementary information [35]. For acoustic loading, $2.5 \mathrm{~nL}$ of droplets can be ejected from a 384-well microtiter plates using an Labcyte Echo acoustic liquid handler (Fig. 1c) [36, 37]. In one setting, droplets are captured by an open port probe (OPP) and then diluted into a continuous solvent flow that enters an ESI source (Fig. 1c) [31, 38, 39]. The combination of precise droplet loading with continuous solvent dilution greatly reduce ion suppression so that chromatography and SPE can be eliminated. When separation steps are omitted, however, cautions should be taken against matrix effects and reduced capability of quantitation.

\section{How MS assays can benefit the engineering of biofuel enzymes}

When summarizing recent engineering studies targeting biofuel-related enzymes (Table 2), it is notable that high-throughput MS assays have not been widely

Table 1 Comparison of various MS platforms in biofuel research

\begin{tabular}{lll}
\hline MS platform & $\begin{array}{l}\text { Analytical time per } \\
\text { sample }\end{array}$ & Biofuel-related analyte \\
\hline GC-MS & $5-20$ min [17] & Limited to small, volatile molecules (short-chain alcohol, fatty acid, hydrocarbon, fatty ester, etc.) \\
LC-ESI-MS & $10-60$ min [14] & Most versatile (Glycan, lipid, fatty acid-derived molecules of various chain lengths) \\
$\begin{array}{l}\text { Automated SPE cleanup } \\
\text { and ESI MS }\end{array}$ & $\sim 10$ s [27, 28] & \\
$\begin{array}{l}\text { Microfluidics-ESI MS } \\
\text { Acoustics-OPP-ESI MS }\end{array}$ & $<1$ s [24, 29, 30] & \\
MALDI MS imaging & $<5$ s [12, 25, 26] & $\begin{array}{l}\text { Preferably macromolecules (glycan, lipid, etc.) and small, nonvolatile molecules (medium- to long- } \\
\text { chain fatty acid-derived molecules, etc.) }\end{array}$ \\
\hline
\end{tabular}


Table 2 Recent studies of protein engineering in biofuel research

\begin{tabular}{|c|c|c|}
\hline Enzyme type & Target property & Screening assay \\
\hline \multicolumn{3}{|l|}{ Biomass deconstruction } \\
\hline Endoglucanase & Activity & Chromogenic surrogate substrate [40] \\
\hline Endoglucanase & Thermostability & Colorimetric assay on polymerization degree [41] \\
\hline Cellobiohydrolase & Activity/thermostability & Colorimetric assay on reducing sugar [42] \\
\hline Beta-glucosidase & Activity & Chromogenic surrogate substrate [43] \\
\hline Beta-glucosidase & Activity & MALDI MS imaging [44] \\
\hline Endoxylanase & Activity & Colorimetric assay on reducing sugar [45] \\
\hline Endoxylanase & Product inhibition & Chromogenic surrogate substrate [46] \\
\hline Endoxylanase & Thermostability & Colorimetric assay on reducing sugar [47] \\
\hline \multicolumn{3}{|l|}{ Substrate utilization } \\
\hline Glucose oxidase & Stability & Fluorescent redox indicator [48] \\
\hline Cellobiose dehydrogenase & Activity & Fluorescent redox indicator [49] \\
\hline Xylose reductase & Cofactor specificity & Colorimetric assay on $\mathrm{NAD}(\mathrm{P}) \mathrm{H}$ consumption $[50,51]$ \\
\hline Xylose transporter & Activity & Genetic biosensor [9] \\
\hline \multicolumn{3}{|l|}{ Product synthesis } \\
\hline P450 fatty acid decarboxylases & Substrate specificity & GC-MS [52] \\
\hline Citramalate synthase & Activity for C3/C4 alcohol production & Synthetic auxotrophy [53] \\
\hline $\begin{array}{l}\text { 2-Isopropylmalate synthase } \\
\text { Ketoisovalerate decarboxylase }\end{array}$ & Substrate specificity towards $\mathrm{C} 5-\mathrm{C} 8$ alcohol production & GC-MS [54] \\
\hline Fatty acid synthase & Product specificity towards C6/C8 fatty acids & GC-MS [55] \\
\hline Thioesterase & Product specificity towards C8/C12 fatty acids & GC-MS [56] \\
\hline Thioesterase & Product specificity towards C8 fatty acid & Synthetic auxotrophy [57] \\
\hline Lipase & Activity/substrate specificity/stability & Chromogenic surrogate substrate [58-60] \\
\hline Lipase & Enantioselectivity & GC [61] \\
\hline
\end{tabular}

utilized in this research area. Currently, most methods convert substrate and/or product concentrations into spectrophotometric signals via assay development. These assays generally rely on the use of chromogenic or fluorogenic surrogate substrates, chemical and biochemical reactions, and genetic biosensors [6]. Therefore, such measurements are indirect and prone to false positive. On the contrary, MS-based enzyme assays enable direct, label-free measurement. This capability allows the use of native substrates of a target enzyme and hence eliminates the need of surrogate substrates in indirect assays. Moreover, thanks to its mass-resolving power, MS can monitor many reaction species simultaneously. This is particularly useful for enzyme specificity engineering that requires differentiation of subtle, structural changes among similar molecules, which is challenging for spectrophotometric assays. To discuss how such unique capabilities can be beneficial in biofuel development, here we compare MS with existing assays in the context of biomass degradation and product synthesis.

Deconstruction of lignocelluloses into fermentable substrates contributes to the main cost in biofuel production. It is hence critical to improve the activity of biomass-degrading enzymes to reduce such cost [38]. Traditionally, cellulolytic enzyme assays often monitor the increase in reducing sugar ends during cellulose depolymerization (Fig. 2a). One colorimetric assay utilizes 3,5-dinitrosalicylic acid (DNS) that reacts stoichiometrically with reducing functional groups to form 3amino-5-nitrosalicylic acid, which exhibits a specific absorption at $540 \mathrm{~nm}$ (Fig. 2b). Based on this principle, the DNS assay is unable to differentiate various oligosaccharide products and hence only reports overall cellulolytic activities. However, it is desirable to screen for both activity and specificity, as the deconstruction of lignocellulosic feedstock generally requires the synergistic action of cellulases with complementary specificities [3].

On the other hand, MALDI MS is widely applied to analyze glycans [62] and capable of differentiating various oligosaccharide products resulted from the hydrolysis of lignocellulosic substrates (Fig. 2c) [63]. MALDI MS imaging has been developed to screen cellulosedegrading enzymes and relevant applications are recently reviewed [12]. Existing approaches often utilize 


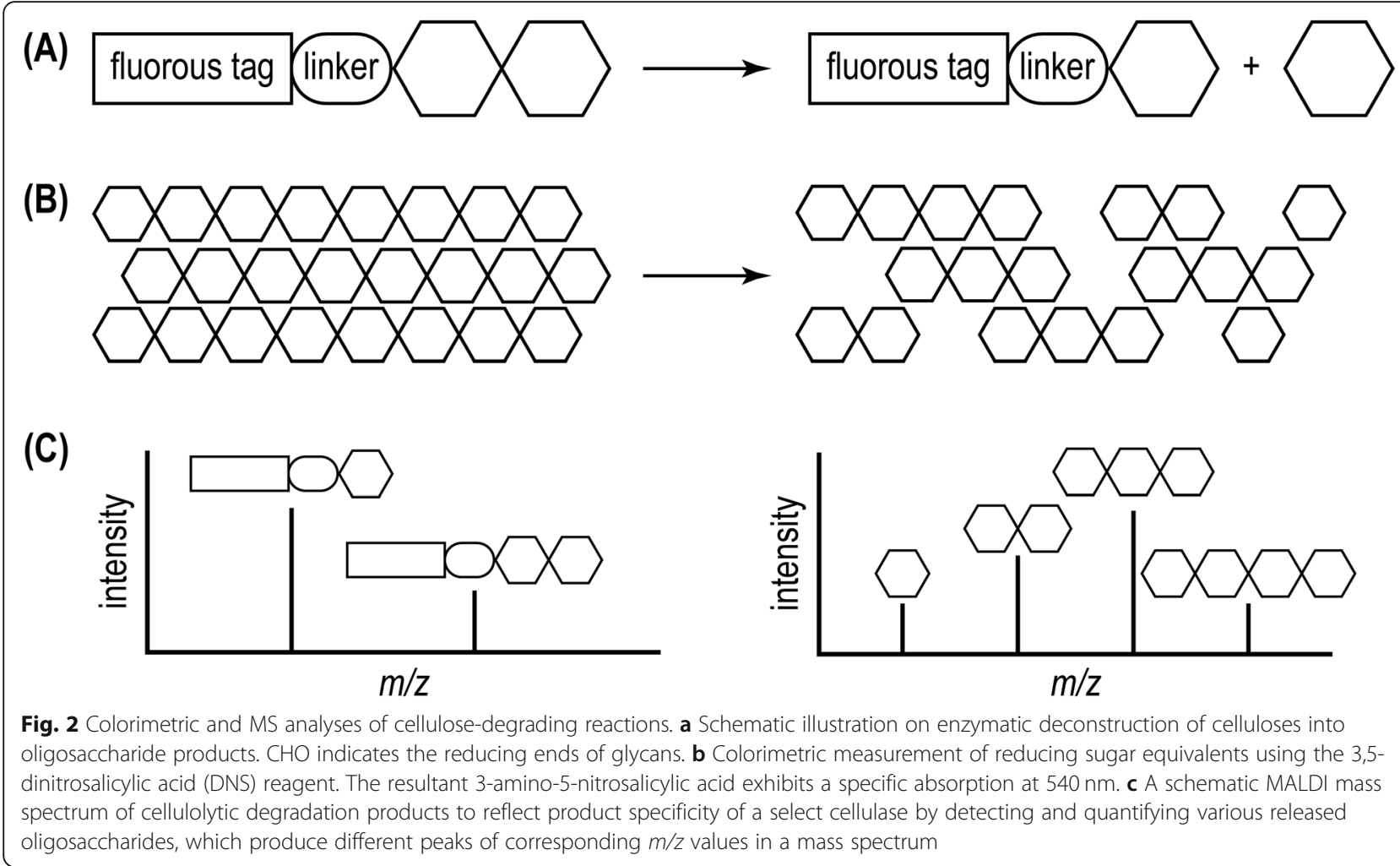

chemically derived substrates that allow covalent or non-covalent immobilization of substrates on a MS target surface [23, 44, 64-66]. For example, perfluorinated glycan analytes can be captured a liquid "initiator" phase on porous silicon surfaces via non-covalent, fluoro-phase interactions. This so-called nanostructureinitiator MS (NIMS) method permits inclusion of washing steps to remove contaminants from complex samples such as cell lysates [65, 66]. Using NIMS, 175 diverse glycosylhydrolases were tested under different temperature and $\mathrm{pH}$ values in microtiter plates, and enzyme reactions were spotted on NIMS chips and analyzed by MALDI MS to generate more than 10,000 data points [44]. An interesting new development combined NIMS with droplet microfluidics, whereby the droplets containing enzyme reaction mixtures were arrayed on discrete NIMS spots at defined time intervals. The subsequent MALDI MS profiling was therefore able to provide time resolved information on the enzyme activities of a glycoside hydrolase [67]. Although effective, the use of chemically derived surrogate substrates may generate screening hits that do not perform well with native substrates. In this regard, it was reported that MALDI MS were used to detect oligosaccharides that were resulted from cellulose and xylan hydrolysis (Fig. 2c) [63]. Therefore, we envision plant biomass can be directly utilized to screen cellulose-degrading enzymes using MALDI MS imaging.
For microbial synthesis of biofuel molecules, it is important to control product composition by engineering enzymes with desirable specificities. For example, fatty acid-derived chemicals with medium chain lengths of 812 are used as "drop-in" fuel alternatives for gasoline, jet fuel, and biodiesel [68]. It has been demonstrated that protein engineering can be applied to alter the specificities of fatty-acid metabolizing enzymes towards medium-chain products $[55,56]$. But it often takes more than $20 \mathrm{~min}$ per sample to analyze the profile of various lipid products using chromatographic separation such as $\mathrm{GC}[55,56]$. To increase screening throughput, chromogenic substrates can be used for colorimetric assays. For example, surrogate ester substrates are utilized for rapid profiling of lipase activities by monitoring the release of $p$-nitrophenol at $405 \mathrm{~nm}$ upon ester hydrolysis (Fig. 3) [58-60]. The specificity of a select lipase can be characterized using a panel of surrogate esters with different fatty acyl chains. However, except for lipases, such chromogenic substrates are not available to most lipidmetabolizing enzymes. In addition, the mutant hits obtained using surrogate substrates do not necessarily perform well with native substrates. Therefore, the lack of high-throughput, generally applicable assays that are specific to fatty acyl chain lengths hinders protein engineering of lipid-metabolizing enzymes [68].

To overcome such limitations, MALDI MS imaging can provide unparalleled speed and selectivity to 


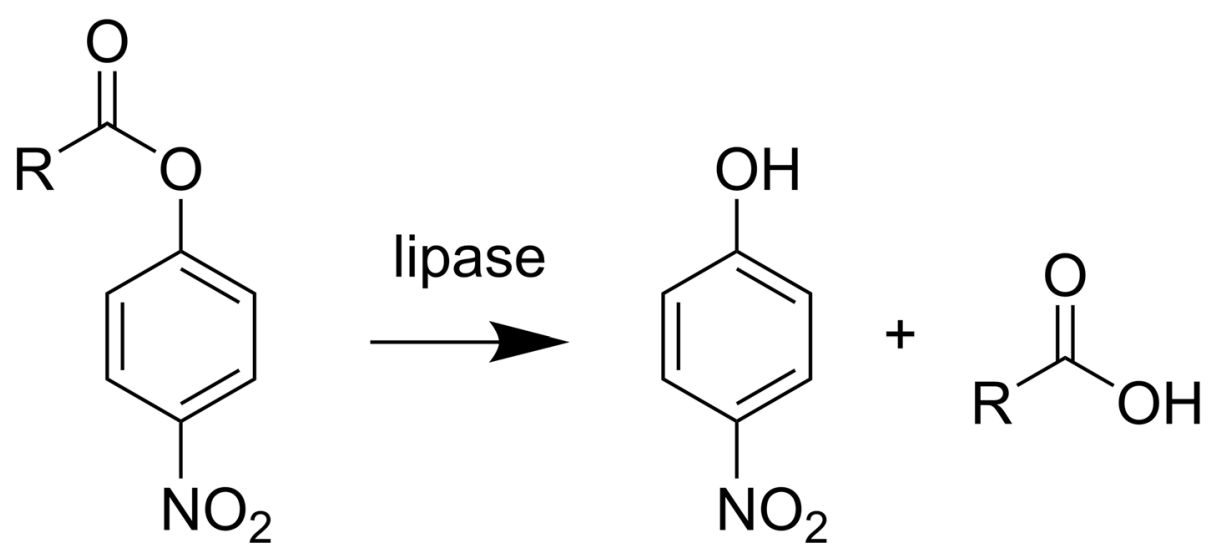

\section{p-nitrophenol}

Fig. 3 Lipase colorimetric assay using surrogate ester substrates. The progress of the lipase reaction can be monitored by measuring the release of $p$-nitrophenol with a specific absorbance at $405 \mathrm{~nm}$

distinguish lipid congeners of various chain lengths based on mass differences. For example, we recently developed optically guided MALDI MS to profile the chemical content of microbial colonies at a rate of $\sim 5 \mathrm{~s}$ [26]. The total and relative abundance of various rhamnolipid congeners were rapidly quantified by monitoring the ion intensities at corresponding $\mathrm{m} / \mathrm{z}$ values (Fig. 4). Using this method, we were able to rapidly screen thousands of mutant strains for directed enzyme evolution [26]. However, biofuel-relevant lipid molecules, including free fatty acids, fatty alcohols, and alkenes, are challenging targets for MALDI MS. The underlying technical difficulties are due to the low molecular weight, low ionization efficiency, and high volatility of these molecules. To overcome these difficulties, assays need be developed to enhance MALDI MS detection, including chemical derivatization [69], the use of nonclassical MALDI matrices such as nanoparticles [70], and detection of easy-to-ionize metabolic precursors such as membrane lipid species [71].

Alternatively, ESI MS assays may also be developed to screen fatty-acid derived products. For example, lipid

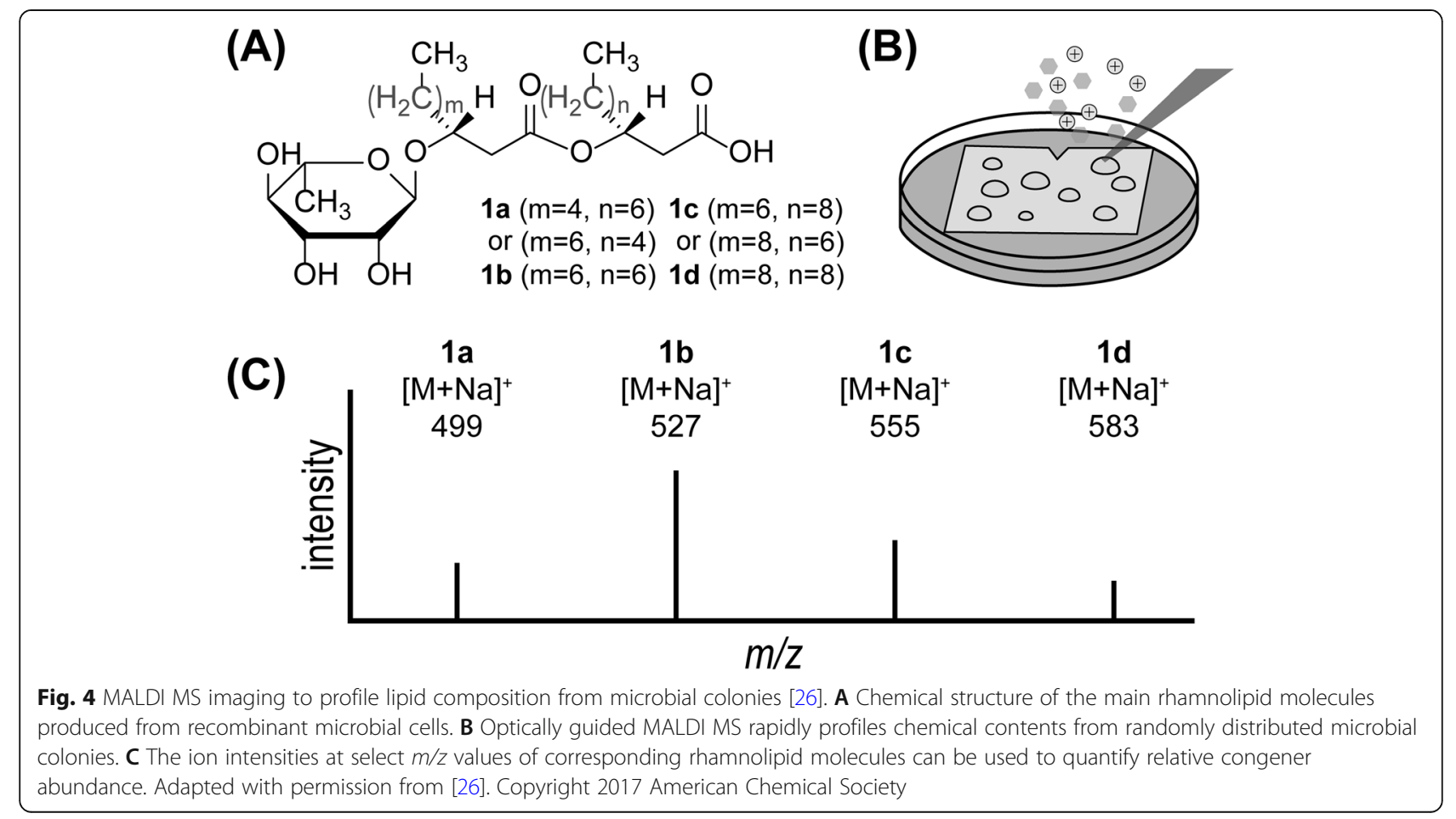


molecules from other biological samples have been analyzed in high throughput through online SPE cleanup using the Agilent RapidFire platform [72]. Acoustic droplet loading from microtiter plates via the OPP-ESI platform should also be applicable. The use of microfluidic droplet-ESI MS settings, however, is not recommended because lipid products may diffuse out of aqueous droplets into surrounding oils due to their hydrophobic nature.

\section{Conclusions}

Here we provided a brief update on new MS platforms for high-throughput enzyme screening in the context of biofuel production. Two main trends are observed. First, it is desirable to eliminate timeconsuming chromatographic separation before MS analysis. In this context, MALDI MS imaging and automated, miniaturized loading to ESI MS are particularly useful. Second, MS is capable of label-free analysis so that native products and industrially relevant conditions can be utilized. This is important for protein engineering because "you get what you screen for" in high-throughput screening. On the other hand, there are foreseeable challenges. Many biofuel molecules are of low polarity and exhibit low ionization efficiency. Moreover, for volatile products such as ethanol, butanol, and medium-chain alkanes, reliable quantitation can be challenging for certain MS types that requires high vacuum (Table 1 ). New advances in instrumentation and sample preparation may help to address the limitation in analyte ranges, such as the development of atmospheric pressure MALDI mass spectrometer [73]. For example, laser-assisted rapid evaporative ionization MS (LA-REIMS) has recently been applied to screen violacein and betulinic acid-producing yeast colonies at a rate of 6 colonies per minute [74]. Moreover, when separation steps are omitted to increase throughput, cautions should be taken against matrix effects and reduced accuracy of quantitation. Therefore, a secondary validation step using GC-MS or LC-MS is still necessary to confirm the positive hits resulted from the primary, highthroughput MS screening. In this regard, it is also of great interest to develop fast chromatographic technologies, such as ultra-high performance liquid chromatography [75], multiplex, overlapping injections in a single run [76, 77], and the simultaneous use of multiple columns in parallel [78]. It is important to select a combination of appropriate MS-based approaches because no single platform provides all chemical information. In addition, as many biofuel researchers may have limited MS experience, it is necessary to develop bioinformatic pipelines that visualize large, complex mass spectral data in a manner similar to classical, colorimetric assays [26]. With continuous endeavor in addressing the abovementioned challenges, we envision a wide application of MS approaches in biofuel enzyme research considering the combined advantages of sensitivity, selectivity, speed, and information-richness for chemical analysis.

\section{Supplementary information}

Supplementary information accompanies this paper at https://doi.org/10. 1186/s42500-020-0011-8.

Additional file 1. Graphical abstract and article highlight.

\section{Abbreviations}

DNS: 3,5-dinitrosalicylic acid; ESI: Electrospray ionization; GC: Gas chromatography; LC: Liquid chromatography; MALDI: Matrix-assisted laser desorption/ionization; MS: Mass spectrometry; NIMS: Nanostructure-initiator mass spectrometry; OPP: Open port probe; QQQ: Triple quadrupole; SPE: Solid-phase extraction; TOF: Time-of-flight

Acknowledgements

Not applicable.

Authors' contributions

TS conceived the scope of this work. LHF, JZZ and TS contributed in drafting, revising, and finalizing the manuscript. All authors approved the submitted version and agreed both to be personally accountable for the author's own contributions and to ensure that questions related to the accuracy or integrity of any part of the work.

\section{Funding}

The authors acknowledge the financial support from Shenzhen Institute of Synthetic Biology, Shenzhen Institutes of Advanced Technology, Chinese Academy of Sciences.

Availability of data and materials

Data sharing not applicable to this article as no datasets were generated or analyzed during the current study.

\section{Competing interests}

The authors declare that they have no competing interests.

\section{Author details}

${ }^{1}$ Institute of Synthetic Biology, Shenzhen Institutes of Advanced Technology, Chinese Academy of Sciences, Shenzhen 518055, People's Republic of China. ${ }^{2}$ Shenzhen Institute of Synthetic Biology, Shenzhen Institutes of Advanced Technology, Chinese Academy of Sciences, Shenzhen 518055, People's Republic of China.

Received: 19 June 2019 Accepted: 30 January 2020

Published online: 21 February 2020

\section{References}

1. Manning HE, Chang J-S, Fang H, Hammond G, Yang Y, Lee SY. BMC energy: a home for all energy and fuels research. BMC Energy. 2019;1(1):1-4. https://doi.org/10.1186/s42500-019-0003-8.

2. Fisher MA, Tullman-Ercek D. Change, exchange, and rearrange: protein engineering for the biotechnological production of fuels, pharmaceuticals, and other chemicals. Curr Opin Biotechnol. 2013;24(6):1010-6. https://doi.org/10.1016/j.copbio.2013.02.027.

3. Wen F, Nair NU, Zhao H. Protein engineering in designing tailored enzymes and microorganisms for biofuels production. Curr Opin Biotechnol. 2009; 20(4):412-9. https://doi.org/10.1016/j.copbio.2009.07.001.

4. Wang M, Si T, Zhao H. Biocatalyst development by directed evolution. Bioresour Technol. 2012;115:117-25. https://doi.org/10.1016/j.biortech.2012. 01.054. 
5. Bornscheuer UT, Hauer B, Jaeger KE, Schwaneberg U. Directed evolution empowered redesign of natural proteins for the sustainable production of chemicals and pharmaceuticals. Angew Chem Int Ed Engl. 2019;58(1):36-40. https://doi.org/10.1002/anie.201812717.

6. Dietrich JA, McKee AE, Keasling JD. High-throughput metabolic engineering: advances in small-molecule screening and selection. Annu Rev Biochem. 2010;79:563-90. https://doi.org/10.1146/annurev-biochem062608-095938.

7. Shapaval V, Brandenburg J, Blomqvist J, Tafintseva V, Passoth V, Sandgren $M$, Kohler A. Biochemical profiling, prediction of total lipid content and fatty acid profile in oleaginous yeasts by FTIR spectroscopy. Biotechnol Biofuels. 2019;12:140. https://doi.org/10.1186/s13068-019-1481-0.

8. He Y, Wang X, Ma B, Xu J. Ramanome technology platform for label-free screening and sorting of microbial cell factories at single-cell resolution. Biotechnol Adv. 2019; 37(6):107388. https:/doi.org/10.1016/j.biotechadv.2019.04.010.

9. Wang M, Li S, Zhao H. Design and engineering of intracellular-metabolitesensing/regulation gene circuits in Saccharomyces cerevisiae. Biotechnol Bioeng. 2016;113(1):206-15. https://doi.org/10.1002/bit.25676.

10. Alvarez-Gonzalez G, Dixon N. Genetically encoded biosensors for lignocellulose valorization. Biotechnol Biofuels. 2019;12:246. https://doi.org/ 10.1186/s13068-019-1585-6.

11. Cheng F, Tang XL, Kardashliev T. Transcription factor-based biosensors in high-throughput screening:advances and applications. Biotechnol J. 2018; 13(7):e1700648. https://doi.org/10.1002/biot.201700648.

12. de Rond T, Danielewicz M, Northen T. High throughput screening of enzyme activity with mass spectrometry imaging. Curr Opin Biotechnol. 2015;31:1-9. https://doi.org/10.1016/j.copbio.2014.07.008.

13. Lv H. Mass spectrometry-based metabolomics towards understanding of gene functions with a diversity of biological contexts. Mass Spectrom Rev. 2013;32(2):118-28. https://doi.org/10.1002/mas.21354.

14. Scheerle RK, Graßmann J. Chapter 10. LC-MS for the Determination of the Enzymatic Activity of Proteins. In: Letzel T, editor. Protein and Peptide Analysis by LC-MS. Cambridge: Royal Soc Chemistry; 2011. p. 133-41.

15. Lee D, Bowen B, Northen T. Mass spectrometry-based metabolomics, analysis of metabolite-protein interactions, and imaging. BioTechniques. 2010:49(2):557-65. https://doi.org/10.2144/000113451.

16. Baran R, Reindl W, Northen TR. Mass spectrometry based metabolomics and enzymatic assays for functional genomics. Curr Opin Microbiol. 2009;12(5): 547-52. https://doi.org/10.1016/j.mib.2009.07.004.

17. Greis KD. Mass spectrometry for enzyme assays and inhibitor screening: an emerging application in pharmaceutical research. Mass Spectrom Rev. 2007; 26(3):324-39. https://doi.org/10.1002/mas.20127.

18. Liesener A, Karst U. Monitoring enzymatic conversions by mass spectrometry: a critical review. Anal Bioanal Chem. 2005;382(7):1451-64. https://doi.org/10.1007/s00216-005-3305-2.

19. Bothner B, Chavez R, Wei J, Strupp C, Phung Q, Schneemann A, Siuzdak G. Monitoring enzyme catalysis with mass spectrometry. J Biol Chem. 2000; 275(18):13455-9. https://doi.org/10.1074/jbc.275.18.13455.

20. de Rond T, Gao J, Zargar A, de Raad M, Cunha J, Northen TR, Keasling JD. A high-throughput mass spectrometric enzyme activity assay enabling the discovery of cytochrome P450 biocatalysts. Angew Chem Int Ed Engl. 2019; 58(30):10114-9. https://doi.org/10.1002/anie.201901782.

21. Bommarius AS, Sohn M, Kang Y, Lee JH, Realff MJ. Protein engineering of cellulases. Curr Opin Biotechnol. 2014;29:139-45. https://doi.org/10.1016/j.copbio.2014.04.007.

22. Chen Z, Zeng AP. Protein engineering approaches to chemical biotechnology. Curr Opin Biotechnol. 2016;42:198-205. https://doi.org/10. 1016/j.copbio.2016.07.007.

23. Bilal M, lqbal HMN, Hu HB, Wang W, Zhang XH. Metabolic engineering and enzyme-mediated processing: a biotechnological venture towards biofuel production-a review. Renew Sust Energ Rev. 2018;82:436-47. https://doi.org/ 10.1016/j.rser.2017.09.070

24. Diefenbach XW, Farasat I, Guetschow ED, Welch CJ, Kennedy RT, Sun S, Moore JC. Enabling biocatalysis by high-throughput protein engineering using droplet microfluidics coupled to mass spectrometry. ACS Omega. 2018;3(2):1498-508. https://doi.org/10.1021/acsomega.7b01973.

25. Lin S, Dikler S, Blincoe WD, Ferguson RD, Sheridan RP, Peng Z, Conway DV Zawatzky K, Wang H, Cernak T, et al. Mapping the dark space of chemical reactions with extended nanomole synthesis and MALDI-TOF MS. Science. 2018;361(6402). https://doi.org/10.1126/science.aar6236.

26. Si T, Li B, Comi TJ, Wu Y, Hu P, Wu Y, Min Y, Mitchell DA, Zhao H, Sweedler $J$. Profiling of microbial colonies for high-throughput engineering of multistep enzymatic reactions via optically guided matrix-assisted laser desorption/ionization mass spectrometry. J Am Chem Soc. 2017;139(36): 12466-73. https://doi.org/10.1021/jacs.7b04641.

27. Lowe DM, Gee M, Haslam C, Leavens B, Christodoulou E, Hissey P, Hardwicke P, Argyrou A, Webster SP, Mole DJ, et al. Lead discovery for human kynurenine 3-monooxygenase by high-throughput rapidFire mass spectrometry. J Biomol Screen. 2014;19(4):508-15. https://doi.org/10.1177/ 1087057113518069

28. Rohman M, Wingfield J. High-throughput screening using mass spectrometry within drug discovery. Methods Mol Biol. 2016;1439:47-63. https://doi.org/10.1007/978-1-4939-3673-1_3.

29. Steyer DJ, Kennedy RT. High-throughput nanoelectrospray ionization-mass spectrometry analysis of microfluidic droplet samples. Anal Chem. 2019; 91(10):6645-51. https://doi.org/10.1021/acs.analchem.9b00571.

30. Ma C, Tan ZL, Lin Y, Han S, Xing X, Zhang C. Gel microdroplet-based highthroughput screening for directed evolution of xylanase-producing Pichia pastoris. J Biosci Bioeng. 2019. https://doi.org/10.1016/j.jbiosc.2019.05.008.

31. Zhang H, Liu C, Liu J, Hua W, Covey T, Ghislain L, Datwani S, Foley T, Janiszewski J, Troutman M, et al. Acoustic-OPP-MS: the next generation bioanalytical platform for drug discovery with ultra-high throughput. In: ASMS Conference on Mass Spectrometry \& Allied Topics; 2019.

32. Rzagalinski I, Volmer DA. Quantification of low molecular weight compounds by MALDI imaging mass spectrometry-a tutorial review. Biochim Biophys Acta. 2017;1865(7):726-39. https://doi.org/10.1016/j. bbapap.2016.12.011.

33. Comi TJ, Neumann EK, Do TD, Sweedler JV. microMS. A python platform for image-guided mass spectrometry profiling. J Am Soc Mass Spectrom. 2017; 28(9):1919-28. https://doi.org/10.1007/s13361-017-1704-1.

34. Agresti JJ, Antipov E, Abate AR, Ahn K, Rowat AC, Baret JC, Marquez M, Klibanov AM, Griffiths AD, Weitz DA. Ultrahigh-throughput screening in drop-based microfluidics for directed evolution. Proc Natl Acad Sci U S A. 2010;107(9):4004-9. https://doi.org/10.1073/pnas.0910781107.

35. Wink K, Mahler L, Beulig JR, Piendl SK, Roth M, Belder D. An integrated chipmass spectrometry and epifluorescence approach for online monitoring of bioactive metabolites from incubated Actinobacteria in picoliter droplets. Anal Bioanal Chem. 2018;410(29):7679-87. https://doi.org/10.1007/s00216-018-1383-1.

36. Sinclair I, Stearns R, Pringle S, Wingfield J, Datwani S, Hall E, Ghislain L, Majlof L, Bachman M. Novel acoustic loading of a mass spectrometer: toward next-generation high-throughput MS screening. J Lab Autom. 2016; 21(1):19-26. https://doi.org/10.1177/2211068215619124.

37. Zhang $\mathrm{H}$. Acoustic dispensing-mass spectrometry: the next high throughput bioanalytical platform for early drug discovery. Bioanalysis. 2017;9(21):161921. https://doi.org/10.4155/bio-2017-4980.

38. Zabed HM, Akter S, Yun J, Zhang G, Awad FN, Qi X, Sahu JN. Recent advances in biological pretreatment of microalgae and lignocellulosic biomass for biofuel production. Renew Sust Energ Rev. 2019;105:105-28. https://doi.org/10.1016/j.rser.2019.01.048.

39. Datwani S, Ghislain LP. System and method for the acoustic loading of an analytical instrument using a continuous flow sampling probe. In: US Patent. vol. US20190157061A1. 2019.

40. Cecchini DA, Pepe O, Pennacchio A, Fagnano M, Faraco V. Directed evolution of the bacterial endo-beta-1,4-glucanase from Streptomyces sp. G12 towards improved catalysts for lignocellulose conversion. AMB Express. 2018;8(1):74. https://doi.org/10.1186/s13568-018-0602-7.

41. Bashirova A, Pramanik S, Volkov P, Rozhkova A, Nemashkalov V, Zorov I, Gusakov A, Sinitsyn A, Schwaneberg U, Davari MD. Disulfide bond engineering of an endoglucanase from Penicillium verruculosum to improve its thermostability. Int J Mol Sci. 2019;20(7). https://doi.org/10.3390/ ijms20071602.

42. Han C, Li W, Hua C, Sun F, Bi P, Wang Q. Enhancement of catalytic activity and thermostability of a thermostable cellobiohydrolase from Chaetomium thermophilum by site-directed mutagenesis. Int J Biol Macromol. 2018;116: 691-7. https://doi.org/10.1016/j.jijbiomac.2018.05.088.

43. Larue K, Melgar M, Martin VJJ. Directed evolution of a fungal $\beta$ glucosidase in Saccharomyces cerevisiae. Biotechnol Biofuels. 2016;9:5266. https://doi.org/10.1186/s13068-016-0470-9.

44. Heins RA, Cheng X, Nath S, Deng K, Bowen BP, Chivian DC, Datta S, Friedland GD, D'Haeseleer P, Wu D, et al. Phylogenomically guided identification of industrially relevant $\mathrm{GH} 1$ beta-glucosidases through DNA synthesis and nanostructure-initiator mass spectrometry. ACS Chem Biol. 2014;9(9):2082-91. https://doi.org/10.1021/cb500244v. 
45. Wang Q, Du W, Weng XY, Liu MQ, Wang JK, Liu JX. Recombination of thermo-alkalistable, high xylooligosaccharides producing endo-xylanase from Thermobifida fusca and expression in Pichia pastoris. Appl Biochem Biotechnol. 2015;175(3):1318-29. https://doi.org/10.1007/s12010-014-1355-7.

46. Hegazy UM, El-Khonezy MI, Shokeer A, Abdel-Ghany SS, Bassuny RI, Barakat AZ, Salama WH, Azouz RAM, Fahmy AS. Revealing of a novel xylose-binding site of Geobacillus stearothermophilus xylanase by directed evolution. J Biochem. 2019;165(2):177-84. https://doi.org/10.1093/jb/mvy092.

47. Irfan M, Gonzalez CF, Raza S, Rafiq M, Hasan F, Khan S, Shah AA Improvement in thermostability of xylanase from Geobacillus thermodenitrificans C5 by site directed mutagenesis. Enzym Microb Technol. 2018;111:38-47. https://doi.org/10.1016/j.enzmictec.2018.01.004.

48. Kovačević $\mathrm{G}$, Ostafe $\mathrm{R}$, Fischer R, Prodanović R. Influence of methionine residue position on oxidative stability of glucose oxidase from Aspergillus niger. Biochem Eng J. 2019;146:143-9. https://doi.org/10.1016/j.bej.2019.03.016.

49. Blažić M, Balaž A, Prodanović O, Popović N, Ostafe R, Fischer R, Prodanović R. Directed evolution of cellobiose dehydrogenase on the surface of yeast cells using resazurin-based fluorescent assay. Appl Sci. 2019;9(7):15. https:// doi.org/10.3390/app9071413.

50. Zhang M, Jiang ST, Zheng Z, Li XJ, Luo SZ, Wu XF. Cloning, expression, and characterization of a novel xylose reductase from Rhizopus oryzae. J Basic Microbiol. 2015;55(7):907-21. https://doi.org/10.1002/jobm.201400786.

51. Liang L, Zhang J, Lin Z. Altering coenzyme specificity of Pichia stipitis xylose reductase by the semi-rational approach CASTing. Microb Cell Factories. 2007:6:36-46. https://doi.org/10.1186/1475-2859-6-36.

52. Jiang Y, Li Z, Wang C, Zhou YJ, Xu H, Li S. Biochemical characterization of three new alpha-olefin-producing P450 fatty acid decarboxylases with a halophilic property. Biotechnol Biofuels. 2019;12:79-92. https://doi.org/10. 1186/s13068-019-1419-6.

53. Atsumi S, Liao JC. Directed evolution of Methanococcus jannaschii citramalate synthase for biosynthesis of 1-propanol and 1-butanol by Escherichia coli. Appl Environ Microbiol. 2008;74(24):7802-8. https://doi.org/ 10.1128/AEM.02046-08.

54. Kechun Z, Sawaya MR, Eisenberg DS, Liao JC. Expanding metabolism for biosynthesis of nonnatural alcohols. Proc Natl Acad Sci U S A. 2008;105(52): 20653-8. https://doi.org/10.1073/pnas.0807157106.

55. Gajewski J, Pavlovic R, Fischer M, Boles E, Grininger M. Engineering fungal de novo fatty acid synthesis for short chain fatty acid production. Nat Commun. 2017:8:14650-7. https://doi.org/10.1038/ncomms14650.

56. Grisewood MJ, Hernandez Lozada NJ, Thoden JB, Gifford NP, Mendez-Perez D, Schoenberger HA, Allan MF, Floy ME, Lai RY, Holden HM, et al. Computational redesign of acyl-ACP thioesterase with improved selectivity toward medium-chain-length fatty acids. ACS Catal. 2017;7(6):3837-49. https://doi.org/10.1021/acscatal.7b00408.

57. Hernandez Lozada NJ, Lai RY, Simmons TR, Thomas KA, Chowdhury R, Maranas CD, Pfleger BF. Highly active C8-acyl-ACP thioesterase variant isolated by a synthetic selection strategy. ACS Synth Biol. 2018;7(9):2205-15. https://doi.org/10.1021/acssynbio.8b00215

58. Zorn K, Oroz-Guinea I, Brundiek H, Dorr M, Bornscheuer UT. Alteration of chain length selectivity of Candida antarctica lipase a by semi-rational design for the enrichment of erucic and gondoic fatty acids. Adv Synth Catal. 2018;360(21):4115-31. https://doi.org/10.1002/adsc.201800889.

59. Yedavalli $P$, Rao NM. Engineering the loops in a lipase for stability in DMSO Protein Eng Des Sel. 2013;26(4):317-24. https://doi.org/10.1093/protein/gzt002.

60. Zhao G, Wang J, Tang Q, Lan D, Wang Y. Improving the catalytic activity and thermostability of MAS1 lipase by alanine substitution. Mol Biotechnol. 2018;60(4):319-28. https://doi.org/10.1007/s12033-018-0062-y.

61. Ema T, Nakano Y, Yoshida D, Kamata S, Sakai T. Redesign of enzyme for improving catalytic activity and enantioselectivity toward poor substrates: manipulation of the transition state. Org Biomol Chem. 2012;10(31):6299308. https://doi.org/10.1039/c2ob25614b.

62. Harvey DJ. Analysis of carbohydrates and glycoconjugates by matrix-assisted laser desorption/ionization mass spectrometry: an update for 2013-2014. Mass Spectrom Rev. 2018;37(4):353-491. https://doi.org/10.1002/mas.21530.

63. Brasseur C, Bauwens J, Tarayre C, Matteotti C, Thonart P, Destain J, Francis F, Haubruge E, Portetelle D, Vandenbol M, et al. MALDI-TOF MS analysis of cellodextrins and xylo-oligosaccharides produced by hindgut homogenates of Reticulitermes santonensis. Molecules. 2014;19(4):4578-94. https://doi.org/ 10.3390/molecules 19044578.

64. Ban L, Pettit N, Li L, Stuparu AD, Cai L, Chen W, Guan W, Han W, Wang PG, Mrksich M. Discovery of glycosyltransferases using carbohydrate arrays and mass spectrometry. Nat Chem Biol. 2012;8(9):769-73. https://doi.org/10. 1038/nchembio.1022.

65. Northen TR, Lee JC, Hoang L, Raymond J, Hwang DR, Yannone SM, Wong $\mathrm{CH}$, Siuzdak G. A nanostructure-initiator mass spectrometry-based enzyme activity assay. Proc Natl Acad Sci U S A. 2008;105(10):3678-83. https://doi. org/10.1073/pnas.0712332105.

66. Deng K, George KW, Reindl W, Keasling JD, Adams PD, Lee TS, Singh AK, Northen TR. Encoding substrates with mass tags to resolve stereospecific reactions using Nimzyme. Rapid Commun Mass Spec. 2012;26(6):611-5. https://doi.org/10.1002/rcm.6134.

67. Heinemann J, Deng K, Shih SC, Gao J, Adams PD, Singh AK, Northen TR. Onchip integration of droplet microfluidics and nanostructure-initiator mass spectrometry for enzyme screening. Lab Chip. 2017;17(2):323-31. https://doi org/10.1039/c6lc01182a.

68. Sarria S, Kruyer NS, Peralta-Yahya P. Microbial synthesis of mediumchain chemicals from renewables. Nat Biotechnol. 2017;35(12):1158-66. https://doi.org/10.1038/nbt.4022.

69. Wu Q, Comi TJ, Li B, Rubakhin SS, Sweedler JV. On-tissue derivatization via electrospray deposition for matrix-assisted laser desorption/ionization mass spectrometry imaging of endogenous fatty acids in rat brain tissues. Anal Chem. 2016;88(11):5988-95. https://doi.org/10.1021/acs.analchem.6b01021.

70. Wu Q, Chu JL, Rubakhin SS, Gillette MU, Sweedler JV. Dopamine-modified $\mathrm{TiO} 2$ monolith-assisted LDI MS imaging for simultaneous localization of small metabolites and lipids in mouse brain tissue with enhanced detection selectivity and sensitivity. Chem Sci. 2017;8(5):3926-38. https://doi.org/10. 1039/c7sc00937b.

71. Si T, Sweedler JV, Zhao H. High-throughput, mass spectrometry-based screening of microbial libraries to produce designer free fatty acids with custom compositions. In: AlChE annual meeting abstract. Pittsburgh; 2018. https://www.aiche.org/conferences/aiche-annual-meeting/2018/proceeding/ paper/63e-high-throughput-mass-spectrometry-based-screening-microbiallibraries-produce-designer-free.

72. Takamiya M, Sakurai M, Teranishi F, Ikeda T, Kamiyama T, Asai A. Lead discovery for mammalian elongation of long chain fatty acids family 6 using a combination of high-throughput fluorescent-based assay and RapidFire mass spectrometry assay. Biochem Biophys Res Commun. 2016;480(4):7216. https://doi.org/10.1016/j.bbrc.2016.10.103.

73. Kiss A, Hopfgartner G. Laser-based methods for the analysis of low molecular weight compounds in biological matrices. Methods. 2016;104: 142-53. https://doi.org/10.1016/j.ymeth.2016.04.017.

74. Gowers GF, Cameron SJS, Perdones-Montero A, Bell D, Chee SM, Kern M, Tew D, Ellis T, Takats Z. Off-colony screening of biosynthetic libraries by rapid laser-enabled mass spectrometry. ACS Synth Biol. 2019. https://doi. org/10.1021/acssynbio.9b00243.

75. De Vos J, Broeckhoven K, Eeltink S. Advances in ultrahigh-pressure liquid chromatography technology and system design. Anal Chem. 2016;88(1): 262-78. https://doi.org/10.1021/acs.analchem.5b04381.

76. Kuehnbaum NL, Kormendi A, Britz-McKibbin P. Multisegment injectioncapillary electrophoresis-mass spectrometry: a high-throughput platform for metabolomics with high data fidelity. Anal Chem. 2013;85(22):10664-9. https://doi.org/10.1021/ac403171u.

77. Schafer W, Wang H, Welch CJ. Multiple-injection high-throughput gas chromatography analysis. J Sep Sci. 2016;39(15):2978-85. https://doi.org/10. 1002/jssc.201600332.

78. de Biasi V, Haskins N, Organ A, Bateman R, Giles K, Jarvis S. High throughput liquid chromatography/mass spectrometric analyses using a novel multiplexed electrospray interface. Rapid Commu Mass Sp. 1999;13(12):1165-8. https://doi. org/10.1002/(sici)1097-0231(19990630)13:12<1165:.aid-rcm638>3.0.co;2-4.

\section{Publisher's Note}

Springer Nature remains neutral with regard to jurisdictional claims in published maps and institutional affiliations. 